\title{
Effect of Aliphatic Diacid Chain Length on Properties of Semiaromatic Copolyamides Based on PA10T and Their Theoretical Study
}

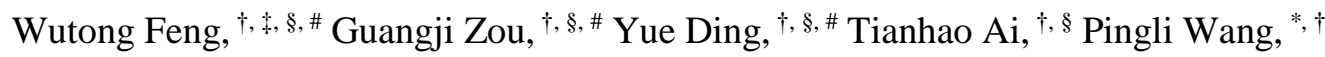 \\ Zhonglai Ren, ${ }^{\dagger}$ and Junhui $\mathrm{Ji}^{*}{ }^{*} \dagger$
}

\begin{abstract}
${ }^{\dagger}$ National Engineering Research Center of Engineering Plastics, Technical Institute of Physics and Chemistry, Chinese Academy of Sciences, Beijing 100190, China

${ }^{\ddagger}$ Shandong Provincial Key Laboratory for Special Silicon-Containing Materials, Advanced Materials Institute, Qilu University of Technology (Shandong Academy of Sciences), Jinan, 250014 China

${ }^{\S}$ University of Chinese Academy of Sciences, Beijing 100049, China
\end{abstract}


1. Chemical structure of the copolyamides

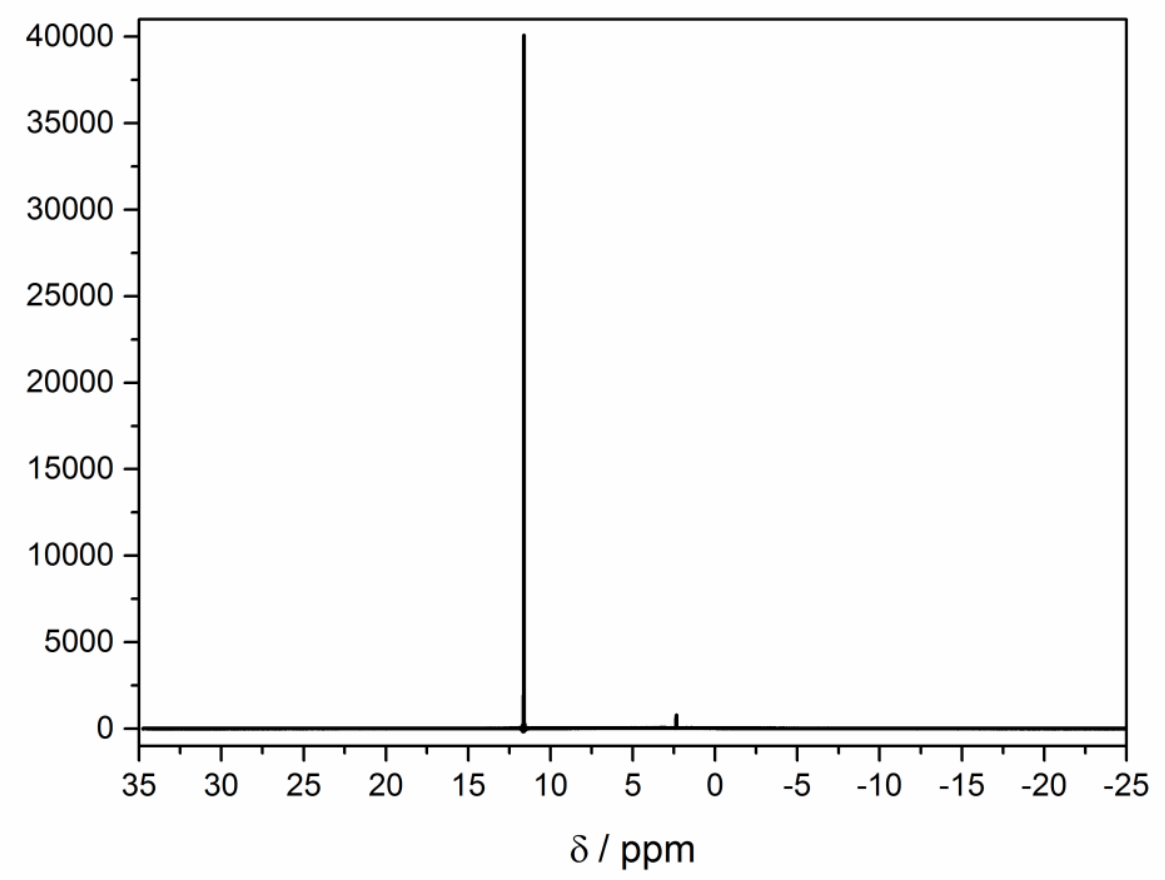

Figure S1. ${ }^{1} \mathrm{H}-\mathrm{NMR}$ spectra of trifluoroacetic acid-D.

Table S1. ${ }^{1} \mathrm{H}-\mathrm{NMR}$ integral data of 10T/10X copolyamides.

\begin{tabular}{cccccccccccc}
\hline & $\delta_{\mathrm{T}}$ & $\delta_{\mathrm{a}}$ & $\delta_{\mathrm{b}}$ & $\delta_{\mathrm{c}}$ & $\delta_{\mathrm{d}}$ & $\delta_{\mathrm{e}}$ & $\delta_{\mathrm{f}}$ & $\delta_{\mathrm{g}}$ & $\delta_{\mathrm{h}}$ & $\delta_{\mathrm{i}}$ & $10 \mathrm{X}$ mol \% \\
\hline S-0 & 1.01 & 2.97 & 1.02 & 1.00 & - & - & - & - & - & & 0 \\
S-1 & 0.90 & 2.93 & 1.13 & 1.00 & 0.11 & - & - & - & - & & 11 \\
S-2 & 0.91 & 3.01 & 1.15 & 1.00 & - & 0.11 & - & - & - & & 11 \\
S-3 & 0.91 & 3.08 & 1.12 & 1.00 & - & - & 0.10 & - & - & & 10 \\
S-4 & 0.90 & 3.16 & 1.11 & 1.00 & - & - & - & 0.10 & - & & 10 \\
S-5 & 0.91 & 3.18 & 1.14 & 1.00 & - & - & - & - & 0.10 & & 10 \\
S-6 & 0.91 & 3.27 & 1.12 & 1.00 & & & & & & 0.10 & 10 \\
\hline
\end{tabular}

2. Binding energy, volume and density of hydrogen bond energy for $10 \mathrm{~T} / 10 \mathrm{X}$ dimers 


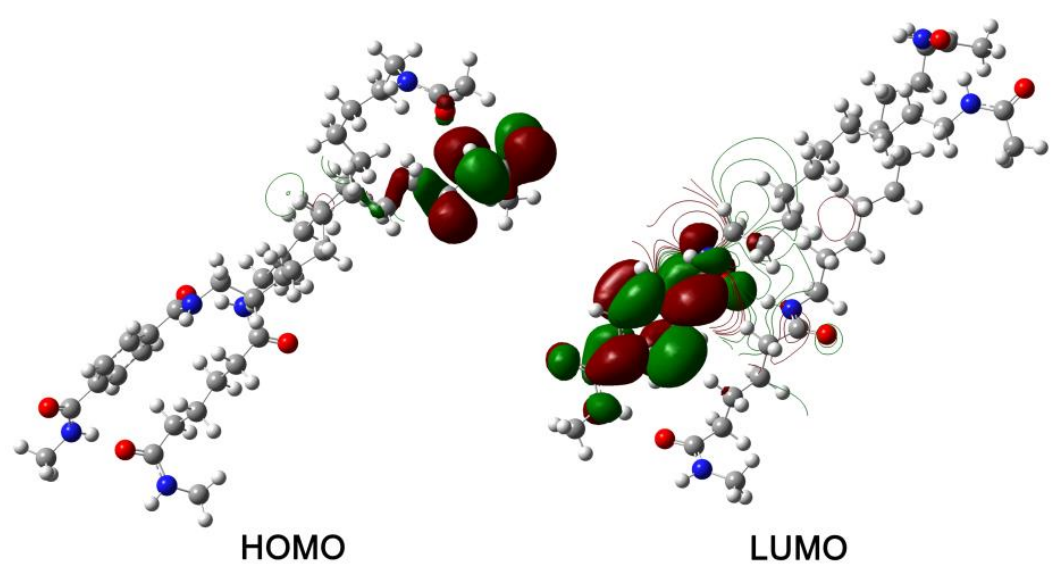

Figure S2. HOMO and LUMO for 10T-106 unit dimers.

\section{Thermal properties of the copolyamides}
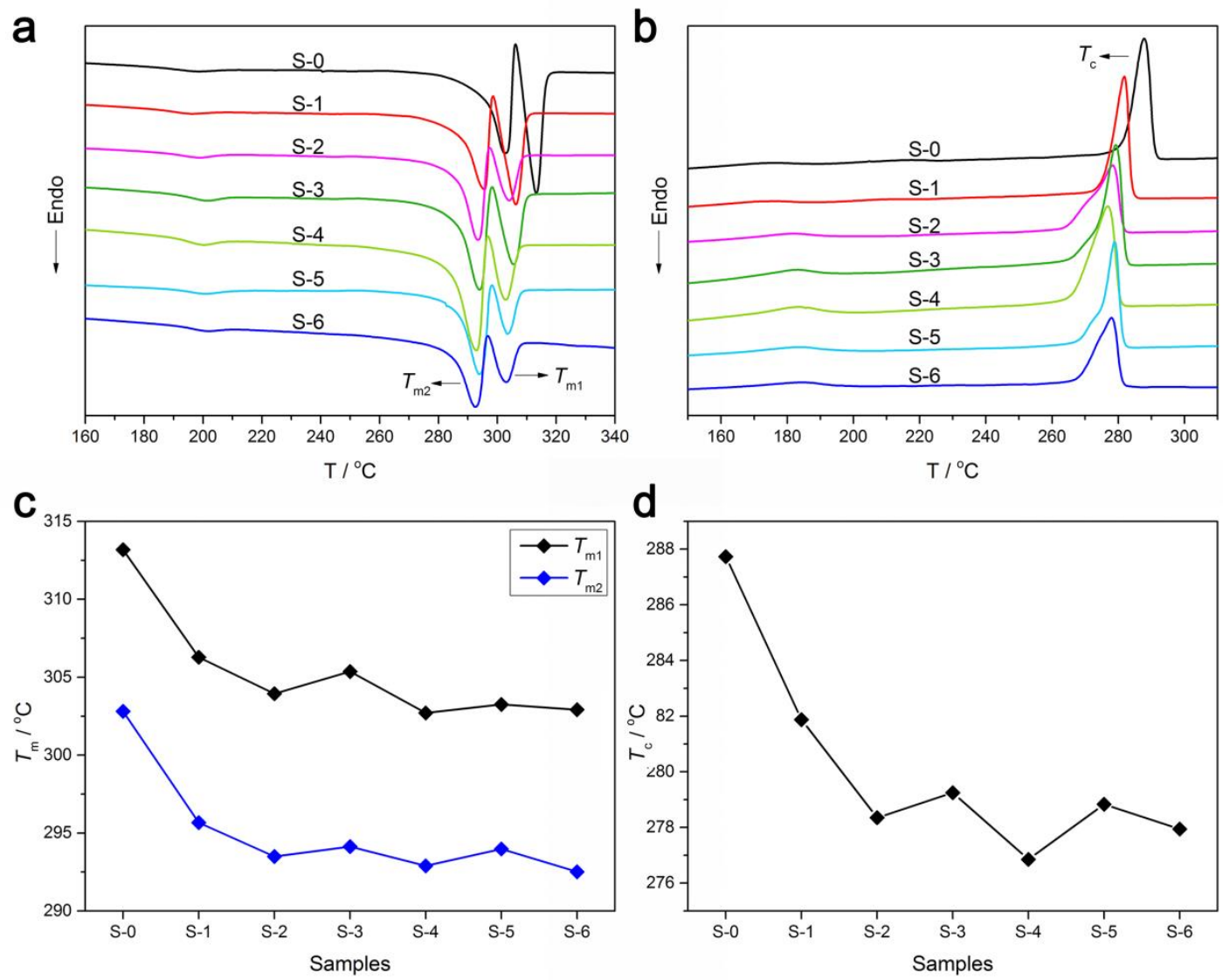

Figure S3. DSC curves of PA10T and 10T/10X copolyamides: (a) heating and (b) cooling curves. The relationship between (c) the melting points, (d) the crystallization temperatures and the samples. 

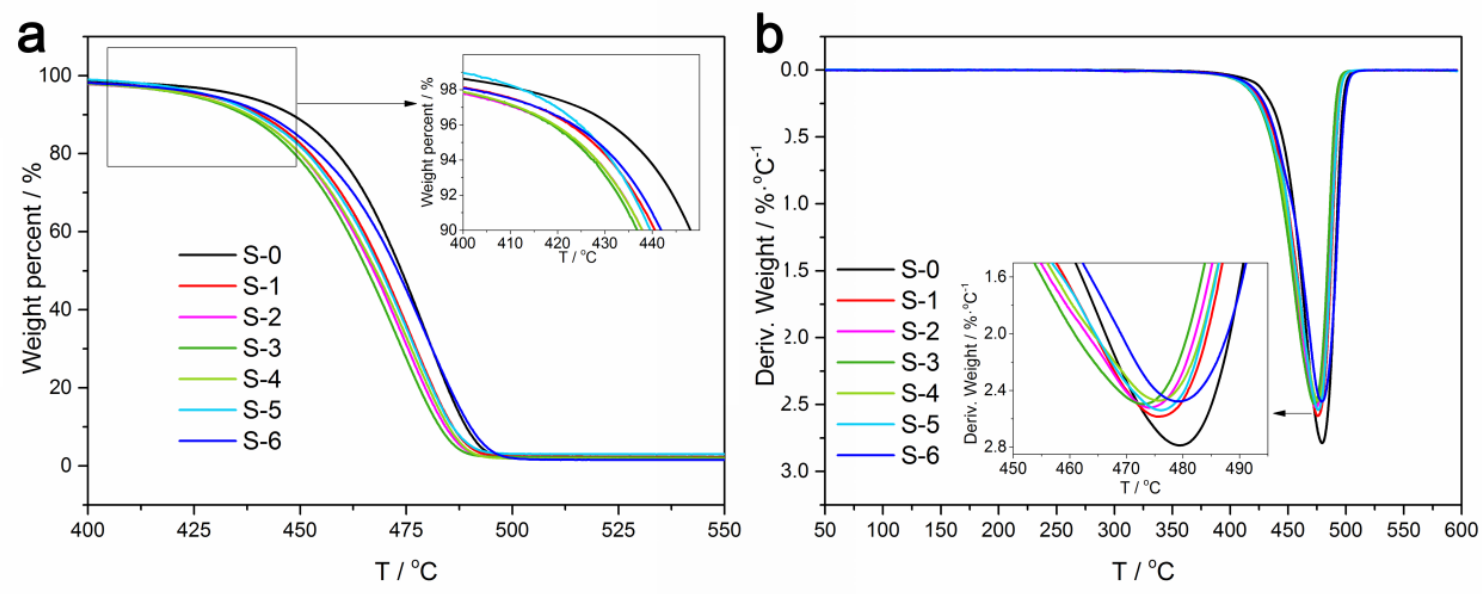

Figure S4. (a) TGA and (b) DTG curves of PA10T and 10T/10X copolyamides.

\section{Mechanical properties of the copolyamides.}

Table S2. Mechanical properties of PA10T and 10T/10X copolyamides.

\begin{tabular}{ccccc}
\hline & $\begin{array}{c}\text { Tensile strength } \\
(\mathrm{MPa})\end{array}$ & $\begin{array}{c}\text { Elongation at break } \\
(\%)\end{array}$ & $\begin{array}{c}\text { Tensile modulus } \\
(\mathrm{MPa})\end{array}$ & $\begin{array}{c}\text { notched impact strength } \\
\left(\mathrm{kJ} / \mathrm{m}^{2}\right)\end{array}$ \\
\hline S-0 & $86.00 \pm 1.85$ & $35.41 \pm 4.28$ & $1459.64 \pm 35.67$ & $13.85 \pm 0.48$ \\
S-1 & $74.40 \pm 1.36$ & $141.50 \pm 22.83$ & $1385.25 \pm 15.87$ & $23.23 \pm 0.18$ \\
S-2 & $73.56 \pm 1.16$ & $227.35 \pm 20.68$ & $1346.23 \pm 9.44$ & $21.56 \pm 0.31$ \\
S-3 & $72.96 \pm 1.81$ & $237.43 \pm 26.07$ & $1339.70 \pm 46.34$ & $19.48 \pm 0.48$ \\
S-4 & $72.64 \pm 1.73$ & $243.23 \pm 23.54$ & $1307.20 \pm 19.55$ & $18.13 \pm 0.31$ \\
S-5 & $72.05 \pm 1.35$ & $249.07 \pm 31.50$ & $1319.91 \pm 12.86$ & $17.19 \pm 0.94$ \\
S-6 & $72.20 \pm 2.56$ & $256.77 \pm 27.82$ & $1268.00 \pm 17.65$ & $16.67 \pm 0.95$ \\
\hline
\end{tabular}

